(c) (i)

Interactive comment on "Offshore and onshore ground-generation airborne wind energy power curve characterization" by Markus Sommerfeld et al.

Markus Sommerfeld et al.

msommerf@uvic.ca

Received and published: 9 June 2021 


\title{
Response to referee 2 wes-2020-120
}

\author{
Markus Sommerfeld
}

Interactive

comment

January 2021

\section{Author response}

Dear referee 2, Thank you very much for your helpful comments to our manuscript, "Offshore and onshore ground-generation airborne wind energy power curve characterization", wes-2020-120. Please accept my apologies for the delayed response.

A lot of time was spend on the revision of this paper including re-clustering WRF wind data, re-running optimizations, re-evaluating results and re-writing major sections of this manuscript. We added a reference section which compares optimization results to quasi steady-state (QSS) AWES and WT reference models. We agree with the criticism to the AWES power coefficient and removed it. Instead, we implemented a brief description and investigation using the harvesting factor?. Please find detailed responses below. I am looking forward to your comments to further improve this paper.

Sincerely, Markus Sommerfeld 


\section{Specific comments}

Line 5 "A universal" instead of "An ..."

- implemented

Interactive

Line 8 annual energy prediction $($ AEP $) \rightarrow$ production

comment

- implemented

Line 249 I'm curious about how pressure \& density vary with stable vs. unstable conditions and how much that affects power.

- That would be interesting to investigate, but was deemed out of scope for this analysis. I would expect the impact on power to be rather small.

Line 271 Why is a reel-out to reel-in ratio used? Is this a combination of a motor torque constraint and the lift during reel-in and reel-out?

- This was a design choice based on conversations with a ground station developer. Motor torque is limited by tether tension.

Line 279 Assumed lift and drag on reel-in and reel-out should be included here

- It is hard to a priori estimate lift and drag as it highly depends on angle of attack, side slip angle and tether drag. Therefore, I would refer to figure 9 which summarizes representative lift, drag and pitch moment coefficients.

Line 280 Was a power constraint used? It's implied in other places.

- Power was indirectly limited by tether speed and tension constraints. 
Line 357 l'd address elevation angle here; based on figure 10, it looks like the optimizer found a common optimal elevation angle for several of the cases, which links tether length and altitude. Vander Lind 2013 calculated an optimal elevation angle for flygensystems assuming an exponential wind profile; I'm curious how close this elevation angle is.

- Brief elevation angle analysis added.

Interactive

comment

Line 398 Missing $U^{3} ?$

- implemented

Line $440 l_{\text {path }}$ and $A_{\text {swept }}$ aren't in table 3

- section removed

Line 459 The fit for $\mathrm{cp}$ is a function of $c_{\text {wing }}$ (and because AR is constant, a function of $A_{\text {swept }}$ ) so it's not non-dimensional and it's not clear how generalizable it is (changes in $A R$ or $L / D$ ). I'm curious about whether another definition of cp may also be comparable to conventional wind turbines but work better. The Loyd paper (see eqs. 1 and 16) shows a limit on a $\mathrm{cp}\left(4 / 27 C L^{3} / C D^{2}\right)$ defined by wing area. What does your data show for a $\mathrm{cp}$ defined by $A_{\text {wing }}$ ? Or if you express $\mathrm{cp}$ as a function of $L / D$ or $C L^{3} / C D^{2}$ ?

- section removed 